\title{
The Sun-Spectroscopic Observations of the Coronal Emission Lines and ADITYA-1 Mission
}

\author{
Jagdev Singh \\ Indian Institute of Astrophysics, Bangalore, India \\ Email: jsingh@iia.res.in
}

\begin{abstract}
The sun and the solar atmosphere, known as solar corona have been studied in detail for centuries but still lot need to be understood about the sun. In the late 19th and earlier 20th century, the information gathered about the solar corona was from the brief moments available during the total solar eclipses. In the later part space observations provided a large body of information about the solar corona. We obtained systematic high resolution spectroscopic observations in [Fe x] 637.4 [Fe xi] 789.2, [Fe xiii] 1074.7, and [Fe xiv] $530.3 \mathrm{~nm}$ emission lines during the period of 1997-2007 with the 25-cm coronagraph at Norikura, Japan. The variation in line widths with height above the solar limb implies that one pair of lines indicate that top of coronal loops are hotter where as other pair of lines shows that loop top is cooler. To explain these results, we proposed an empirical model as all these results are difficult to explain using the existing models. With this background we planned and designed a visible emission line coronagraph (VELC). This instrument is in advance stage of fabrication and is expected to be launched in 2019-20. The challenges and required technical development for this mission are discussed.
\end{abstract}

Keywords: ADITYA-1 Mission, Solar corona, VELC,

\section{Introduction}

The sun is nearest star to earth at a distance of $\sim 150$ million Km from the earth. Sun's core temperature is about 14 million degrees whereas surface temperature is only $5700 \mathrm{~K}$ degrees. The surface of the sun shows features as sunspots, filaments, prominences, active regions around sunspots known as plages etc. and these vary with 11-year period. The amplitude of the variations also varies from one cycle to another cycle. Apart from the above mentioned features one sees granulation and super-granulation on the solar surface which have average life time of about 10 minutes and 20 hours, respectively. The average size $(30,000 \mathrm{Km})$ of super-granules was found to be varying with the phase of solar cycles (Singh and Bappu, 1981). Apart from the 11-year solar cycle variations there are quasi-periodic variations with long periods. Just after the invention of the telescope by Galileo in 1910 and discovery of sunspots on the sun, sunspots did not appear on the sun for about 60-70 years during the period 1645-1715). This period is known as Maunder Minimum. The observations of the sun as star shows that the sun's brightness varies by about 0.2 percent in the continuum (Photosphere); by $\sim 30 \%$ in $\mathrm{Ca}-\mathrm{K}$ line (Chromosphere) and $\sim 100$ to $300 \%$ in X-rays (solar corona) with the solar cycle being more at the maximum phase of the sun.

Before 1940, the scientist thought that atmosphere of the sun is cooler than the surface temperature. It was, only in early 1940's, realised that atmosphere is much hotter than the surface temperature when the green emission line discovered during the total solar eclipse of August 7, 1969 was 
attributed to [Fe xiv] by Edlen (1943). The observations of many coronal emission lines indicate that temperature of the solar corona is 1-2 million degrees and is inhomogeneous. The solar corona can be observed during the brief moment of total solar eclipse, with coronagraphs situated at high altitudes during clear blue skies. The scattering of sun light due to the atmosphere of the earth limits the view of corona to 4 solar radii even during the total solar eclipse but corona can be observed up to large distances with coronagraph in the space above the earth's atmosphere. Because of high temperature of the solar corona, it can be observed in EUV, and X-rays against the solar disk as sun is very poor emitter in $\mathrm{X}$ rays with telescopes in space.

How the coronal plasma gets heated up to millions of degrees, is still not well understood? It is generally believed that magnetic energy is responsible to heat up the coronal plasma but actual processes taking place need to be investigated. Coronal plasma can be heated up through the existence of various types of waves or / and by small and large scale reconnections of flux tubes. With a view to understand the heating of coronal plasma, we made systematic spectrographic observations of the coronal emission lines, 4 lines simultaneously during the period 19972007 using 25-cm coronagraph at Norikura observatory of National Astronomical

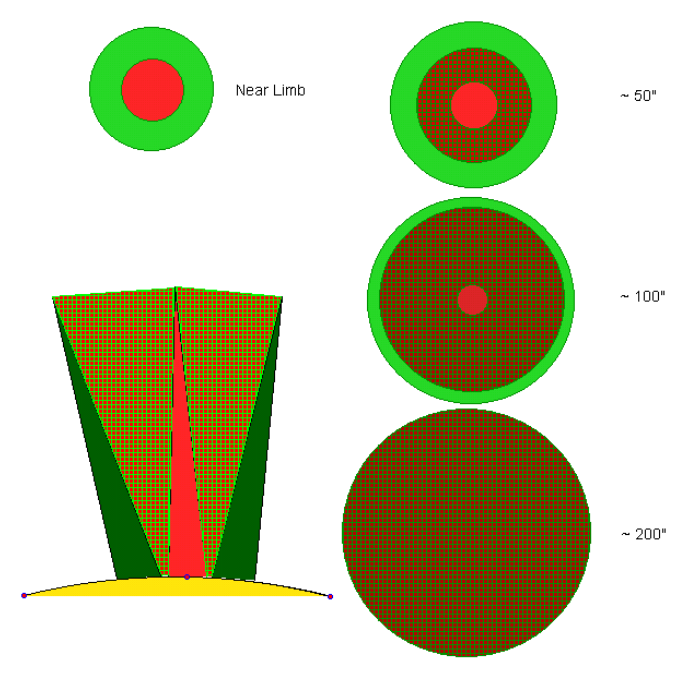

Figure 1 (Color online): 3-D contour map of the proposed empirical model to explain all the findings. Yellow represents the photosphere. Red (cooler) and green (hotter) area represents coronal plasma in a loop (in the plan of sky) above the solar limb. The circles represent cross-section perpendicular to the loop near the limb, at 50, 100 and 200 arcsec above the limb.

\section{Observatory of Japan}

We found that width of the red emission line at $637.4 \mathrm{~nm}$ due to $[\mathrm{Fe} \mathrm{x}]$ representing the coronal plasma at a temperature of $\sim 1 \mathrm{MK}$ increases with height above the solar limb whereas the width of the green line at 530.3 $\mathrm{nm}$ due to [Fe xiv] representing plasma at a temperature of $\sim 1.8 \mathrm{MK}$ decreases with height above the solar limb (Singh et al. 1999, 2002, 2003, 2004, 2006a, 2006b). It is surprising since heating of coronal plasma or /and increase in the non-thermal of the plasma due to the wave propagation should increase the width of both the emission lines with height above the limb. Further, we found that intensity ratio [Fe xi]/ [Fe $\mathrm{x}]$ of emission lines increased with height above the limb whereas intensity ratio of [Fe xiv] / [Fe xiii] decreased with height above the limb. It is unexpected 
result as it indicates that coronal loop tops are hotter as compared to foot-points of the loop if these are observed in [Fe xi] and [Fe x] emission lines and loop tops are cooler if these are observed in [Fe xiv] and [Fe xiii] emission lines.

\section{Theoretical Models and Proposed Empirical Model}

There are two types of models to explain the plasma in the solar corona. One assumes the existence of various kinds of waves (MHD waves etc.) in the solar corona those gets dissipated and heat the corona, the other is spontaneous heating of plasma by small scale and large reconnection of magnetic field which are observed as small and large solar flares. The occurrence of these events lead to change in the emission line profiles in those coronal regions. The existing theoretical models, assume magnetic pressure to be very high as compared to the gas pressure in the coronal loops, mostly isothermal in nature and magnetically shielded different temperature loops, can explain observations of one emission line at a time by assuming increase in non-thermal velocity with height due to the existence of coronal waves (increase in line width) or dissipation of waves in the solar corona (decrease) in line width with height). But the models can't explain the observed variations in

line-width and intensity ratios with height in all the four emission lines simultaneously.

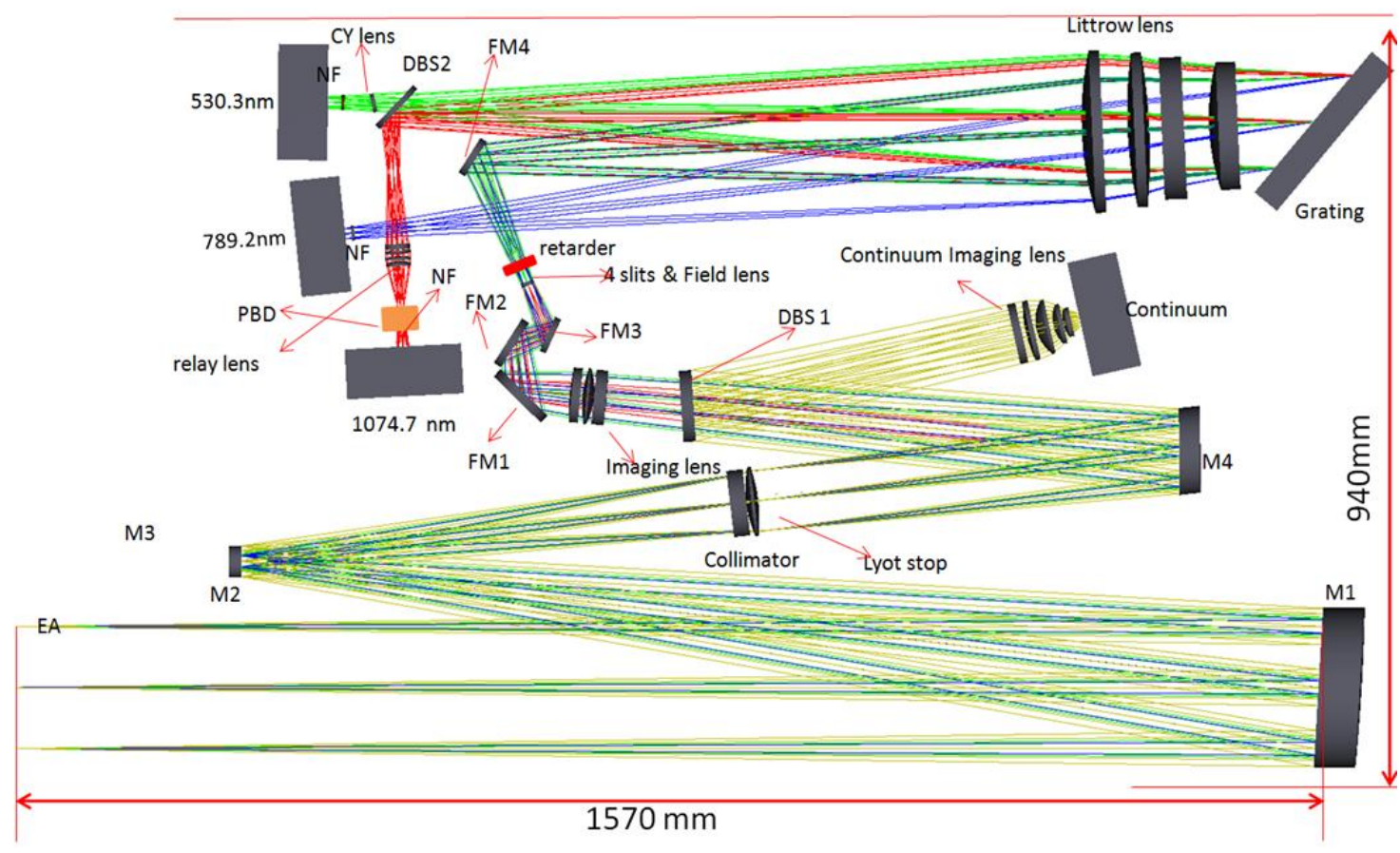

Figure 2 (Color online): Optical layout of VELC to take the images of the solar corona in continuum at $490 \mathrm{~nm}$ and perform spectroscopy.

We, therefore, proposed an empirical model to explain all the results of all the emission lines observed simultaneously. In this model 
we assume that broad structure of the coronal loop is associated with the active region at the base of coronal loop at the photosphere / chromosphere which lives for few hours to few days. In the broad loop there are thin

strands of loop which are due to plasma at different temperature and relatively high density. These have short life and formed due to spontaneous heating of plasma by small scale reconnection at the base of coronal loop. At the base of coronal loop where the magnetic field is relatively stronger, the different temperature plasma remains separate but starts interacting with each other when it moves above the limb as the magnetic field weakens. The general structure of coronal loop indicates high temperature plasma (green area) surrounds relatively cooler plasma (red area) as shown in the contour map of Figure 1 near the foot-points of a coronal loop. At higher heights above the limb the neighbouring different temperature plasma starts interacting with each other and this process goes on increasing with increasing height. The interaction between different temperature plasma leads to sharing of energy, thereby cooler plasma becomes relatively hotter and hotter plasma becomes relatively cooler. Our large amount of obtained on many days of many coronal loops indicate that at $\sim 200$ arcsec above the limb coronal plasma attains uniform common temperature and non-thermal velocity. The value of common temperature and height of common temperature is likely to vary for different loops depending upon the associated magnitude and topology of magnetic field at the base of the loop at the photospheric level. This way the model explains increase in the line-width the red line as the plasma contributing to this emission becomes hotter with height above the limb and decrease in line-width of green line as plasma contributing to this emission becomes cooler with height above the solar limb. This also explains the increase in intensity ratio of $[\mathrm{Fe}$ $\mathrm{xi}]$ / $[\mathrm{Fe} \mathrm{x}]$ with lesser magnitude than expected considering temperature dependence of ion abundances and decrease in intensity ratio of [Fe xiv] / [Fe xiii] with height above the solar limb.

\section{Visible Emission Line Coronagraph (VELC) on ADITYA-1 mission}

The 25-cm coronagraph at Norikura observatory permitted observations in three emission lines [Fe $\mathrm{x}],[\mathrm{Fe} \mathrm{xiii}]$ and [Fe xiv], simultaneously. The $[\mathrm{Fe} \mathrm{x}]$ could be replaced by [Fe xi] emission lines. But observation in all the 4 emission lines [Fe $\mathrm{x}],[\mathrm{Fe} \mathrm{xi}],[\mathrm{Fe}$ xiii] and [Fe xiv], simultaneously, is very important and necessary to understand the complete nature of the coronal loops. I, therefore, designed and proposed VELC (Singh et al, 2011) to perform the spectroscopic observations in all the 4 emission lines, simultaneously. Due weight restrictions imposed on the payload the observations are planned in 3 emission lines [Fe xi], [Fe xiii] and [Fe xiv] only. The payload will be capable to take the images of solar corona in the continuum at $490 \mathrm{~nm}$ between 1.05 to 3 solar radii, perform spectroscopy of the solar corona in these 3 emission lines up to 1.5 solar radii and take spectro-polarimetric observations using $[\mathrm{Fe}$ xiii] emission line at $1074.7 \mathrm{~nm}$. The optical layout of the instrument is shown in Figure 2. The M1 off axis paraboloid mirror of 1300 $\mathrm{mm}$ focal length receives the sun and coronal light through a $147 \mathrm{~mm}$ entrance aperture which focuses the image sun and corona on M2 mirror. The sun light escapes to the sky 
through a $\sim 13 \mathrm{~mm}$ hole in the M2 mirror whereas coronal light is reflected and directed to a collimator and Lyot-stop to make the beam parallel and remove scattered component due to entrance aperture. Image of the solar corona at $490 \mathrm{~nm}$ is made on a camera with CMOS detector using Dichroic filter (DBS1), Interference filter and a lens system capable of forming diffraction limited images. The rest of the coronal light $>490 \mathrm{~nm}$ is fed to the multi-slit spectrograph with Littrow system. The grating with 600 lines per $\mathrm{mm}$, blazed at angle of $42^{\circ}$ maximises the efficiency of the spectrograph at the required wavelengths, namely 789.2, 1074.7 and 530.3 $\mathrm{nm}$. By keeping retarder and analyser in the coronal beam at the respective places shown in Figure 2 we can also take spectropolarimetric observations to study the coronal magnetic field. The details of optical and mechanical designs have been worked out. Thermal analysis has been done and various issues have been addressed. Laboratory model of the payload to address the issues related to the integration procedure has been made. The satellite with VELC and other instruments is expected to be launched 2019-20.

\section{References}

Edlen, B., 1943, Z. Astrophys., 22,30.

Prasad,K. S., Singh, Jagdev and Ichimoto, K, 2013, ApJ, 765L, 46.

Singh, Jagdev and Bappu, M.K.V., 1981, Solar Phys., 71, 161.

Singh, Jagdev, K.Ichimoto, H.Imai, T.Sakurai and A.Takeda, 1999, PASJ, 51, 269.

Singh, Jagdev, T.Sakurai, K.Ichimoto, Y.Suematsu and A.Takeda, 2002, PASJ, 54, 793.

Singh, Jagdev, K.Ichimoto, T.Sakurai and S.Muneer, 2003, ApJ, 585, 516.

Singh, Jagdev et al. 2004, ApJL, 617, 81L.
Singh, Jagdev et al. 2006a, Solar Phys., 236, 245.

Singh, Jagdev et al. 2006b, ApJ, 639, 475.

Singh, Jagdev and 32 co-authors, 2011, Current Science, 100, 167.

$* * * * * * *$ 
Vol. 14 (1), (2019) 21-25 